\title{
Mechanisms of Wave Transformation in Finite-Depth Water
}

\author{
O. H. Shemdin, ${ }^{\prime}$ S. V. Hsiao, ${ }^{\prime}$ H. E. Carlson, ${ }^{2}$ \\ K. HasselmanN, ${ }^{3}$ AND K. SChUlze ${ }^{4}$
}

\begin{abstract}
Mechanisms of wave transformation in finite-depth water are investigated. The linear mechanisms examined are percolation, bottom motion, shoaling, and refraction. The nonlinear mechanisms examined are wave-wave interaction and bottom friction. New exact computations of the nonlinear transfer for $f$ nite-depth waves are presented for some directional wave spectra. These mechanisms are found to explain satisfactorily wave decay observations obtained at several sites with different bottom sediment properties. The decay rates at these sites are found to be dominated by different mechanisms which are determined by the bottom conditions. As an example, detailed calculations are presented for data obtained at the Jonswap site.
\end{abstract}

\section{INTRODUCTION}

The various data sets reported in the literature on wave transformation in finite-depth water suggest that different mechanisms may be operating at different sites depending on depth and on bottom sedimentary properties. A survey of available data sets on wave height spectra acquired in different water depths and from different physiographic settings was recently reported by Shemdin et al. [1978]. The survey also discussed the various possible mechanisms that impact wave transformation in finite-depth water. It was concluded that both linear mechanisms, such as percolation, bottom motion, shoaling, and refraction, and nonlinear mechanisms, such as wave-wave interaction and bottom friction, can play important roles in energy transfer to or from shoaling waves. A detailed investigation of the bottom motion mechanism was subsequently given by Hsiao and Shemdin [1980]. Similarly, a detailed description of the wave-wave interaction mechanism was provided by Herterich and Hasselmann [1980].

From the investigations referred to above, it is possible to present a summary of the linear and the nonlinear mechanisms which can dominate wave transformation in finitedepth water as shown below:

\section{a. Linear Mechanisms}

Three linear mechanisms can affect the transformation of waves in finite-depth water in addition to wave refraction and shoaling. These are prcolation, bottom motion, and the scattering of waves by bottom irregularities. The last process represents a quadratic interaction between the wave field and the bottom irregularity, but is linear in the wave field itself. These are discussed in the following paragraphs.

Percolation. This mechanism was investigated first by Putnum [1949] for an isotropic sand layer. Kajiura and Reid [1957] reconsidered a more comprehensive version of the problem. The wave pressure field induces a flow in the sand below, which is dependent on the sand permeability. The wave energy dissipation can be computed from the cross correlation between the wave-induced pressure above the bed and the vertical velocity at the top of the bed. It was shown by Shem-

\footnotetext{
I Jet Propulsion Laboratory, California Institute of Technology, Pasadena, California 91103.

${ }^{2}$ Deutsches Hydrographisches Institut, Hamburg, West Germany.

${ }^{3}$ Max-Planck-Institut für Meteorologie, Hamburg, West Germany.

4 University of Hamburg, Hamburg, West Germany.

Copyright $(9) 1980$ by the American Geophysical Union.
}

din et al. [1978] that the rate of wave energy dissipation for a water depth $h$ is given by

$$
\frac{\partial E}{\partial t}=-E k \sqrt{\alpha \beta} \frac{\tanh \sqrt{(\alpha / \beta) k d}}{\cosh ^{2} k h}
$$

where $\alpha$ and $\beta$ are the horizontal and the vertical permeability coefficients, respectively, and $k$ is the wave number. For an isotrophic sand $(\alpha=\beta)$ and a deep sand layer $(d>0.3$ wave length) (1) becomes

$$
\partial E / \partial t=-E k \alpha / \cosh ^{2} k h
$$

which is identical to Putnam's solution to within a constant.

Using laboratory experiments, Sleath [1970] provided coefficients of permeability for a coarse sand (mean diameter $D_{m}$ $=1.3 \mathrm{~mm}$ with standard deviation $=0.46 \mathrm{~mm}$ ) and a fine sand (mean diameter $=0.38 \mathrm{~mm}$, with standard deviation $=0.10$ $\mathrm{mm})$. The calculated $\alpha$ values for the coarse and the fine sands are 1.26 and 0.145 , respectively, and the calculated $\beta$ values are 0.89 and 0.126 , respectively. Expressed in terms of the damping coefficient, defined by

$$
\gamma_{p}=-\frac{1}{E} \frac{\partial E}{\partial t}
$$

one obtains $\gamma_{p}=O\left(10^{-3}\right) / \mathrm{s}$ for coarse sand and $\gamma_{p}=O\left(10^{-4}\right) /$ $s$ for fine sand.

Wave-induced bottom motion. When the bottom sediment is composed of mud or of decomposed organic matter, the bottom responds to the wave-induced pressure field in a viscoelastic manner. This was confirmed in observations reported by Tubman and Suhayda [1976] and by Rosenthal [1978]. A solution for a two-layer coupled-flow model was given by Hsiao and Shemdin [1980] for a finite-depth mud layer. It was shown that the rate of wave energy dissipation owing to bottom motion to be

$$
\frac{\partial E}{\partial t}=-2 k_{,} V_{g} E
$$

where the complex wave number $k$ is specified by

$$
k=k_{r}+i k_{i}=\frac{\omega^{2} 1+\tanh (k h) \Omega}{g \tanh (k h)+\Omega}
$$

$\Omega$ is a complex function of water and mud properties as specified by Hsiao and Shemdin [1980] and $V_{g}$ is the wave group velocity.

Bottom scattering. This mechanism was explored by Long 
[1973] who found it to be potentially important but strongly dependent on the spectrum of bottom irregularities. In the Jonswap area, the measurements of the bottom irregularities by Richter et al. [1976] suggest that this mechanism is inadequate for explaining the observed swell attenuation. The bottom scattering mechanism predicts increased wave energy of offshore propagating waves in the offshore direction. In contrast, other dissipation mechanisms, such as bottom friction or percolation, predict a decrease in wave energy of such waves in the offshore direction. A conclusive verification of this mechanism requires accurate measurements of swell in the onshore and the offshore directions at a minimum of two stations.

\section{b. Nonlinear Mechanisms}

The dominant nonlinear dissipative mechanism in the finite-depth water is bottom friction. In addition, conservative nonlinear wave-wave interactions can play a significant role in the redistribution of wave energy within the wave spectrum. These are discussed in the following paragraphs.

Bottom friction. This mechanism is perhaps the most explored of all bottom-interaction processes. Bottom friction is generally dominant over other dissipative processes when the sediment is composed of sand with mean diameter in the range $0.1-0.4 \mathrm{~mm}$ where low permeability prohibits percolation and granular friction prevents viscous flow behavior. The bottom friction coefficient $C_{f}$ defined as

$$
\tau_{b}=C_{f} \rho\left|\mathbf{U}_{b}\right| \mathbf{U}_{b}
$$

where $\tau_{b}$ is the bottom shear stress, $\rho$ is the water density, and $\mathbf{U}_{b}$ is the orbital velocity just outside the bottom boundary layer, depends on sand ripple characteristics as reported by Jonsson [1965], Tunstall and Inman [1975], Dingler [1974], and Kamphuis [1975]. The concept of a friction diagram was introduced by Jonsson [1965] for calculating the friction coefficient under various bottom roughness conditions. Hsiao and Shemdin [1978] demonstrated that this concept is useful in explaining the varied wave decay behavior in finite-depth waters. It was found that observed variability in decay rates was linked to variability in the friction coefficient. The latter could be calculated from the properties of the sediment at a site and the prevailing sea-state conditions acting at the site.

Nonlinear wave-wave energy transfer. The nonlinear energy transfer owing to third-order wave-wave interactions is given by a Boltzman integral. Herterich and Hasselmann [1980] computed the transfer rate in finite-depth water for a narrowpeak spectrum, for which the interactions are concentrated in a limited region around the peak wave number $\mathbf{k}_{p}$ by using a modification of the approximate technique of Longuet-Higgins [1976] and Fox [1976]. Their results indicate that for $k_{p} h$ $\leqslant O(1)$, the nonlinear wave-wave energy transfer is strongly dependent on $k_{p} h$. The magnitude increases dramatically for $k_{p} h \leq 0.5$, suggesting the critical importance of this mechanism in wave transformation in shallow water. For very shallow water regions such as in the surf zone, the assumption of weakly nonlinear interaction fails, and the results given by Herterich and Hasselmann [1980] no longer pertain. As is in all processes discussed above, the calculations are valid in a shelf region bound by deep water on one side and by the surf zone on the other.

In this paper we present in section 2 new exact computations on the nonlinear wave-wave transfer for finite-depth waves for directional wave spectra. We then consider in sec- tion 3 a radiative transfer model for calculating wave transformation in finite-depth water which takes into account the various mechanisms discussed. In section 4 we present an example of detailed calculations for data obtained in the Jonswap site. Finally, we summarize in section 5 the relative importance of various mechanisms affecting wave transformation in finite-depth water.

\section{SOME EXACT CoMpUtations ON NONLINEAR TRANSFER IN FinITE-DEPTH WAVES}

The nonlinear energy transfer owing to third-order wavewave interactions is given by a Boltzman integral shown below

$$
\begin{aligned}
& \frac{\partial F}{\partial t}\left(\mathbf{k}_{4}, h\right)=\int \pi \omega_{4}\left(\frac{3 g^{2} D}{2 \omega_{1} \omega_{2} \omega_{3} \omega_{4}}\right)^{2} \\
& \cdot\left[\omega_{4} F_{1} F_{2} F_{3}+\omega_{3} F_{1} F_{2} F_{4}-\omega_{1} F_{2} F_{3} F_{4}-\omega_{2} F_{1} F_{3} F_{4}\right] \\
& \delta\left(\mathbf{k}_{1}+\mathbf{k}_{2}-\mathbf{k}_{3}-\mathbf{k}_{4}\right) \cdot \delta\left(\omega_{1}+\omega_{2}-\omega_{3}-\omega_{4}\right) d \mathbf{k}_{1} d \mathbf{k}_{2} d \mathbf{k}_{3}
\end{aligned}
$$

which describes the rate of change of the spectrum at wave number $k_{4}$ owing to all interactions with other components $k_{1}$, $k_{2}, k_{3}$ which satisfy the resonance conditions

$$
\begin{aligned}
& k_{1}+k_{2}=k_{3}+k_{4} \\
& \omega_{1}+\omega_{2}=\omega_{3}+\omega_{4}
\end{aligned}
$$

The depth dependence in (6) enters through the nonlinear interaction coefficient $D$ and the dispersion relationship, particularly in the frequency $\delta$ function. Exact computations of the energy transfer in (6) were performed numerically by $\mathrm{Hassel}$ mann and Hasselmann [1980] in finite-depth water for directionally varied spectra. These results are shown in Figures 1 and 2. Figure 1 shows the shapes of Jonswap directional spectrum used for the nonlinear transfer calculations. Here, $\theta$ corresponds to direction of wave propagation.

Figure $2 a$ shows the two-dimensional nonlinear transfer rate of the spectrum shown in Figure 1 for $k_{p} h=0.5$, where $k_{p}$

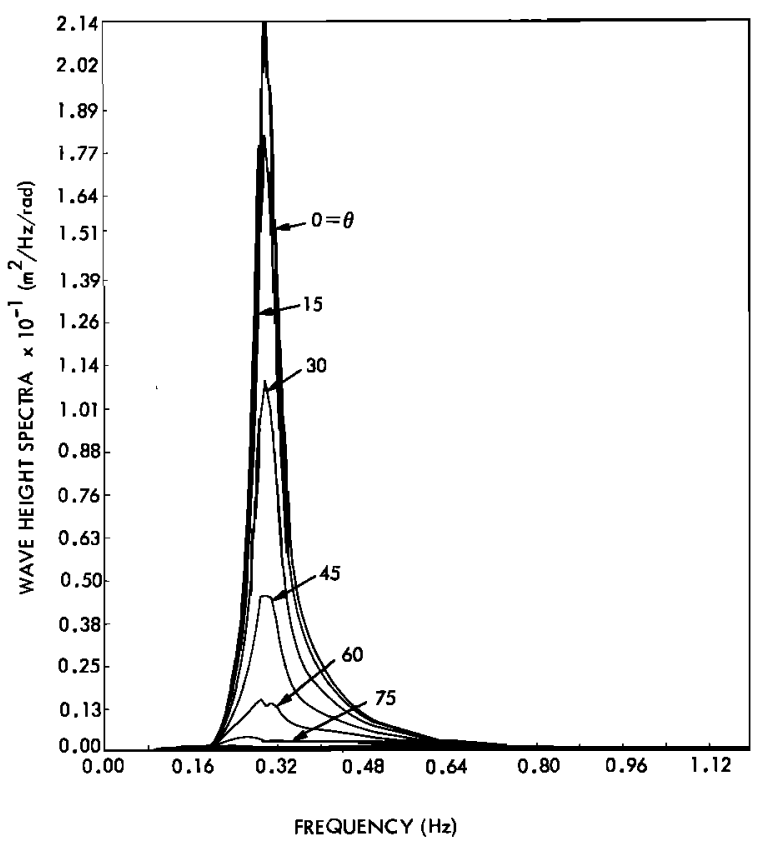

Fig. 1. Graphical display of the Jonswap directional wave height spectrum. Significant wave height, $H_{s}=0.6 \mathrm{~m}$. 


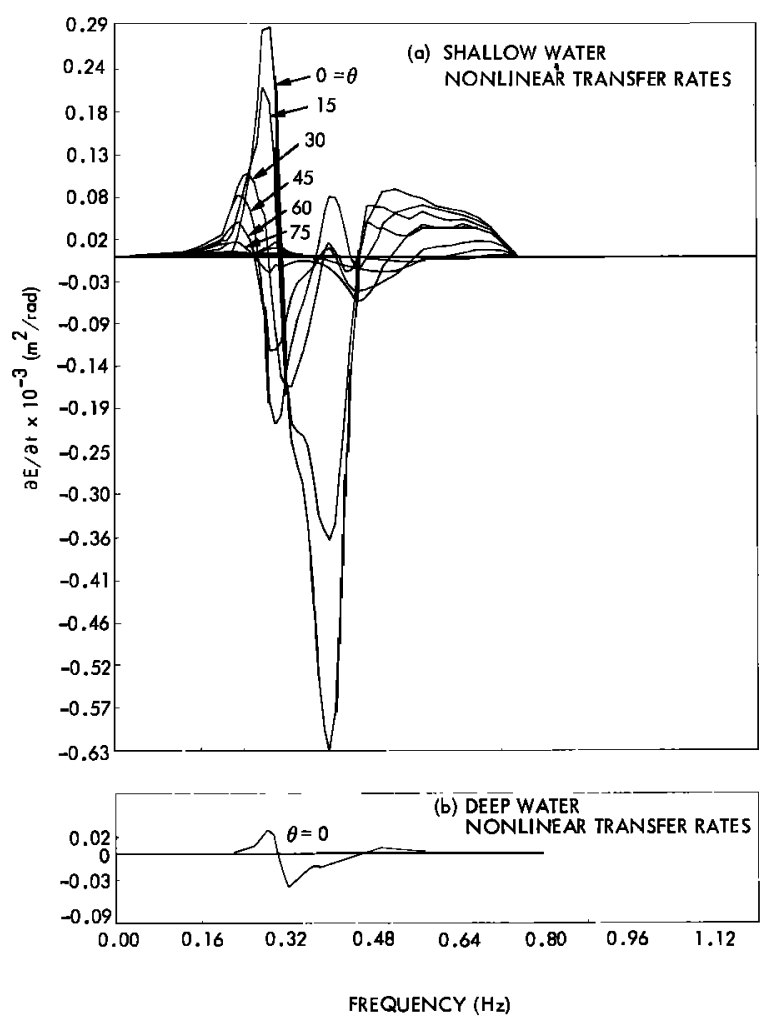

Fig. 2. (a) Nonlinear wave-wave energy transfer in finite-depth water for directional wave height spectrum shown in Figure 1. (b) Deep water nonlinear wave-wave transfer for $\theta=0$.

is the dominant wave number and $h$ is the water depth. For comparison, the transfer rate for the same spectrum in deep water is shown in Figure $2 b$ when $\theta=0$. Note that the transfer rate in finite-depth water is 1 order of magnitude greater than that in deep water. The ratio of the transfer rate in shallow water to that in deep water under otherwise equivalent conditions is shown in Figure 3 as a function of $k_{p} h$. The points denoted by circles are the computations by Hasselmann and Hasselmann [1980], and the solid curve is the theoretical value for a narrow spectrum proposed by Herterich and Hasselmann [1980]. The rapid increase in this ratio with decreasing $k_{p} h$ outlines the importance of the nonlinear transfer mechanism in finite-depth water.

\section{Radiative Transfer Model for Finite-DePThs}

The radiative transfer equation for the wave spectrum $F(x$, $\mathbf{k} ; t$ ) has the form

$$
\frac{\partial F}{\partial t}+\dot{x}_{i} \frac{\partial F}{\partial x_{i}}+\dot{k}_{i} \frac{\partial F}{\partial k_{i}}=S
$$

where

$$
\begin{aligned}
& \dot{x}_{i}=\frac{\partial \omega}{\partial k_{\imath}}(\mathbf{x}, \mathbf{k} ; t)=V_{g} \\
& \dot{k}_{\imath}=-\frac{\partial \omega}{\partial x_{i}}(\mathbf{x}, \mathbf{k} ; t)
\end{aligned}
$$

Here $V_{g l}$ is the group velocity, $\omega$ is wave frequency, and $S$ is the net source function. The latter can be divided further into the various linear and nonlinear components

$$
S=S_{p}+S_{b}+S_{f}+S_{n}+S_{s}
$$

The first three refer to energy dissipation by percolation, bottom motion, and bottom friction, respectively. The last two, $S_{n}$ and $S_{s}$, refer to energy transfer by nonlinear wave-wave interaction and bottom scattering, respectively.

Here (7) applies in the absence of currents (in the presence of currents, the energy spectrum should be replaced by the action spectrum $F / \omega^{\prime}$, where $\omega^{\prime}$ is the intrinsic wave frequency in the reference frame moving with the current). In a one-dimensional steady wave field in which the depth contours are straight and parallel, (7) simplifies to the form

$$
\frac{\partial \omega}{\partial k_{x}} \frac{\partial F}{\partial x}-\frac{\partial \omega}{\partial x} \frac{\partial F}{\partial k_{x}}=S
$$

In terms of the directional frequency spectrum $E(f, \theta)$ defined by

$$
E(f, \theta) d f d \theta=F(\mathbf{k}) d \mathbf{k}
$$

where $f$ is the wave frequency and $\theta$ propagation direction, or, with

$$
\begin{aligned}
\frac{d \mathbf{k}}{d f d \theta} & =\frac{2 \pi k}{V_{g}} \\
E & =\left(2 \pi k / V_{g}\right) F
\end{aligned}
$$

It can be shown that (11) takes the form

$$
V_{g} \cos \theta \frac{d}{d x}\left(\frac{E V_{g}}{2 \pi k}\right)=S
$$

Here $\theta$ is defined as the angle between the wave normal and the $x$ axis normal to the depth contours. The rate of change of $E$ in the direction normal to shore may then be expressed as

$$
\frac{d E}{d x}=-\left(\frac{1}{k} \frac{d k}{d x}+\frac{1}{V_{g}} \frac{d V_{g}}{d x}\right) E+\frac{2 \pi k}{V_{g}^{2} \cos \theta} S
$$

The first and second terms on the right-hand side of (15) represent the contributions from wave refraction and shoaling, respectively. The last term represents the total change in energy owing to dissipation or nonlinear energy transfer.

Each of the dissipation mechanisms can now be incorporated in (15) by specifying the forms of the contributions of $S$ as listed in (10). Thus the source function for percolation is given by

$$
S_{p}=-\frac{V_{g} E}{2 \pi} \sqrt{\alpha \beta} \frac{\tanh \sqrt{(\alpha / \beta) k d}}{\cosh ^{2} k h}
$$

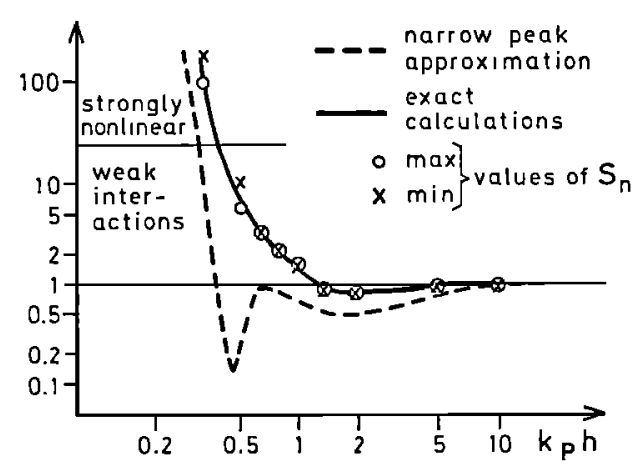

Fig. 3. Ratio $R^{\prime}$ of the nonlinear transfer for finite-depth waves to the nonlinear transfer for deep water waves. Open dots denote determined from the exact computation. Solid curve denotes inferred by Herterich and Hasselmann [1980] for a narrow peak spectra. 
Similarly, $S_{b}$ has the form (see (4))

$$
S_{b}=-\frac{1}{\pi} \frac{k_{s}}{k} \frac{E}{\cos \theta}
$$

Appropriate representations for $S_{f}$ were given by Hasselmann and Collins [1968], using a full spectral formulation, and Bretschneider and Reid [1954] for a narrow spectral peak.

The transfer rate owing to bottom scattering $S_{s}$ was evaluated by Long [1973]. The importance of this mechanism is not established conclusively yet. Consequently, it was not included in the radiative transfer calculations in this paper. Finally, the nonlinear wave-wave interaction transfer rate $S_{n}$ was calculated by using the results given in (2).

If the depth contours are not straight or parallel, (7) cannot be reduced to a one-dimensional equation such as (15). In this case a far more complicated two-dimensional equation has to be solved, in which the rays of all wave components have to be computed at all grid points, and all rays are coupled through the nonlinear mechanisms.

\section{Detailed Calculations of Wave TRANSFORMATION AT THE JONSWAP SITE}

As was indicated previously, the relative importance of the various dissipative mechanisms at a site is strongly dependent on the sediment mean grain size diameter. Percolation is most effective in coarse sand $\left(D_{m} \geq 0.5 \mathrm{~mm}\right)$ [see Savage, 1953]. In finer sand $\left(D_{m}<0.4 \mathrm{~mm}\right)$, bottom friction becomes more important than percolation. The friction coefficient also is critically dependent on the presence or absence of stable sand ripples on the bottom. Friction may even dominate over percolation in coarse sand if sand ripples are present. Finally, when the bottom is composed of silt, clay, or soft organic matter, bottom motion can become dominant in dissipating wave energy.

In the Jonswap area the swell decay rates were calculated by Hasselmann et al. [1973] from wave spectra at different offshore stations. The decay rate $\Gamma$ was defined as

$$
\Gamma=d \ln I / d \xi
$$

where $I$ is the wave energy in the swell band and $\xi$ is a normalized nonlinear distance from shore that incorporates the shoaling properties of waves. The functional form of decay rate parameter, $\Gamma$, depends on the energy dissipation mechanisms at work. The nonlinear friction mechanism requires $\Gamma$ to have the form

$$
\Gamma=g C_{f} V_{g}
$$

where $C_{f}$ is the friction coefficient. The linear percolation mechanism requires $\Gamma$ to have the form (see (1)-(3))

$$
\Gamma=\left[\frac{\omega^{2} \cosh ^{2}(k h)}{k^{2}}\right] \gamma_{p}
$$

A preliminary examination of sediment conditions in the Jonswap area (K. Richter, private communication, 1980) revealed the existence of medium to coarse sand on the bottom in the area south of the Jonswap line and patches of fine sand in the area north of this line. The presence of coarse sand can be expected in an active ocean environment such as the Jonswap area. The dissipation rates $\Gamma$ were calculated by using the percolation mechanism along the Jonswap line for sand with a mean grain diameter of $1.0 \mathrm{~mm}$ and a wave frequency of $0.09 \mathrm{~Hz}$ (most frequently observed in the reported data).
The $\Gamma$ values in 10 and $20 \mathrm{~m}$ water depths were calculated to be 0.032 and 0.080 , respectively, using the relationship between $\Gamma$ and $\gamma_{p}$. The calculated $\Gamma$ values bracket the mean value derived from the measurements $(0.05)$ and therefore support the hypothesis that the percolation mechanism is an important one along the Jonswap line.

In contrast to the above, the bottom motion mechanism for this area predicts $\Gamma$ values to be 1 order of magnitude less than the mean value derived from measurements [Rosenthal, 1978]. We next consider detailed spectral calculations for the area north of the Jonswap line where medium to fine sand (mean grain diameter $0.17-0.3 \mathrm{~mm}$ ) was observed to be present (K. Richter, private communication, 1980). Two cases of wave transformation in finite-depth water are computed for the area between station $8 \mathrm{~N}(10 \mathrm{~km}$ NNE of station 8$)$ and station $5 \mathrm{~N}$ (10 km NNE of station 5 ) of the Jonswap profile. Station 8 is located $27 \mathrm{~km}$ offshore of Sylt at coordinate point $\left(55^{\circ} 04.9^{\prime} \mathrm{N}, 7^{\circ} 57.2^{\prime} \mathrm{E}\right)$. The water depth is $17 \mathrm{~m}$. Station 5 is located $9.5 \mathrm{~km}$ offshore at coordinate point $\left(5502.2^{\prime} \mathrm{N}, 8^{\circ}\right.$ $12.7^{\prime} \mathrm{E}$ ). Here the water depth is $13 \mathrm{~m}$. The two cases considered are for wave spectra measured on August 23, 1975, at (1) $1200 \mathrm{GMT}$ and (2) $2000 \mathrm{GMT}$, respectively. Wave heights were measured at both stations $8 \mathrm{~N}$ and $5 \mathrm{~N}$ with wave rider buoys. In addition, directional wave height measurements were obtained with a pitch-and-roll buoy at station 8 . The latter was used to determine the directional differences between wind and waves at station 8 . Wind speed and direction measurements were available at station 8 , at the meteorological station at List, and at the Land station during the period of interest. For the first case the dominant waves at station 8 were found to be propagating from direction $320^{\circ}$ from true north, and the wind measured at station 8 was $7.0 \mathrm{~m} / \mathrm{s}$ from $310^{\circ}$. For the second case the dominant waves at station 8 were also from $320^{\circ}$ true north, and the wind was $9.0 \mathrm{~m} / \mathrm{s}$ from $300^{\circ}$ true north.

The results of the computations for case (1) are shown in Figure 4. In Figure $4 a$ the measured spectra at station $8 \mathrm{~N}$ (significant wave height, $\left.H_{s}=1.5 \mathrm{~m}\right)$ and at station $5 \mathrm{~N}\left(H_{s}=1.3\right.$ m) are shown. Also the computed spectrum at station $5 \mathrm{~N}\left(H_{s}\right.$ $=1.3 \mathrm{~m}$ ), using the measured spectrum at station $8 \mathrm{~N}$ as input, is shown for comparison. The agreement is reasonable for the dominant frequencies in the spectrum. At the high-frequency end, however, the measured spectrum does not show the energy gain owing to atmospheric input shown in the computed spectrum. In Figure $4 b$ the computed differences in wave energy between stations $8 \mathrm{~N}$ and $5 \mathrm{~N}$ are shown at each wave frequency. The total change in energy as well as the contributions from each of the energy transfer mechanisms are delineated. The results here indicate that for this case the wave energy change is dominated by friction and to a lesser extent by shoaling and refraction. Because $k_{p} h$ values for this case varied between 0.96 and 1.10 (see Figure 3 ) the nonlinear wave-wave transfer was relatively small and of the same order as that occurring in deep water. The atmospheric transfer to waves here is found to be only appreciable at frequencies greater than $0.25 \mathrm{~Hz}$ and is offset by near-surface wave energy dissipation owing to turbulence, possible interaction with long waves, and wave breaking. The latter mechanism is being actively investigated by Longuet-Higgins [1978].

Case 1 represents a typical wave height spectrum of the Jonswap type which has one dominant peak. In case 2 we perform calculations on a spectrum with two dominant frequency peaks as shown in Figure 5. The type of computations per- 

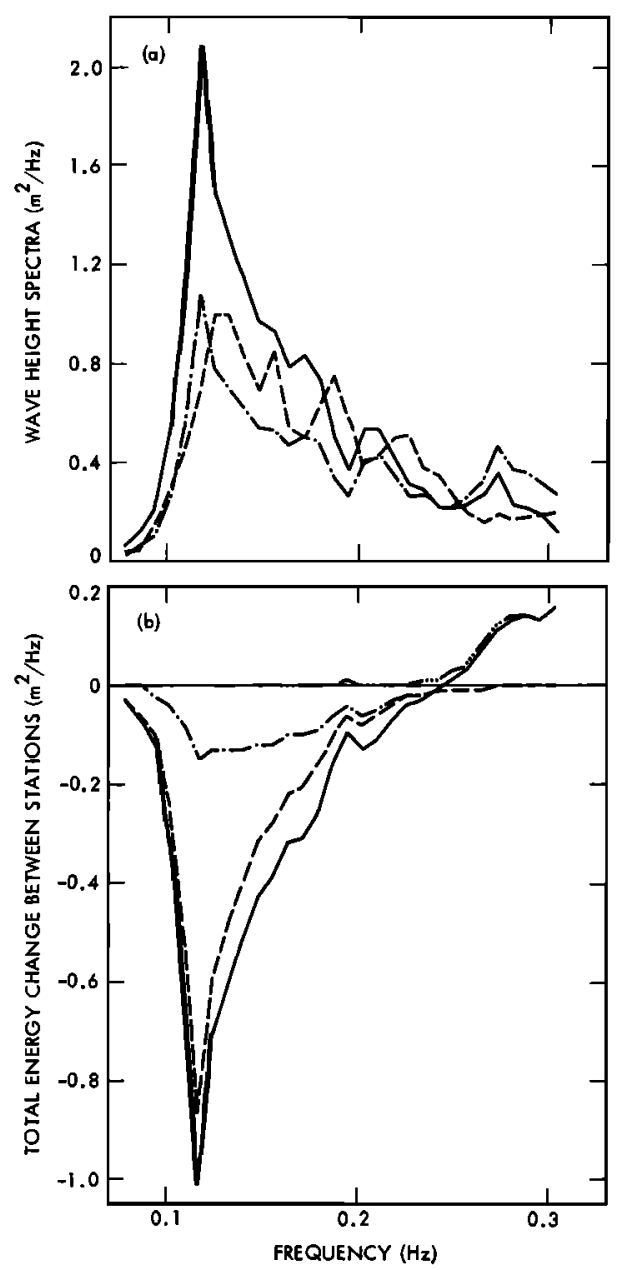

Fig. 4. Wave transformation computations in finite-depth water for case 1, August 23, 1975 at 1200 GMT. (a) Solid curve denotes measured spectrum at station $8 \mathrm{~N}\left(H_{s}=1.5 \mathrm{~m}\right)$, dashed curve denotes measured spectrum at station $5 \mathrm{~N}\left(H_{s}=1.3 \mathrm{~m}\right)$, and dashed-dotted curve denotes computed spectrum at station $5 \mathrm{~N}$ by using measured station $8 \mathrm{~N}$ spectrum as input $\left(H_{s}=1.3 \mathrm{~m}\right)$. (b) Total computed energy change between stations $8 \mathrm{~N}$ and $5 \mathrm{~N}$. Solid curve denotes total change, dashed curve denotes frictional dissipation component, dashed-dotted curve denotes refraction and shoaling component, dashed double dotted curve denotes nonlinear wave-wave transfer component, and dashed triple dotted curve denotes atmospheric input component.

formed for this case as well as station designations are identical to those for case 1 shown in Figure 4. The results are also similar. In Figure $5 a$ the computed spectrum at station $5 \mathrm{~N}\left(H_{s}\right.$ $=1.3 \mathrm{~m}$ ) is in reasonable agreement with that measured at station $5 \mathrm{~N}\left(H_{s}=1.2 \mathrm{~m}\right)$ near the dominant frequencies but differs for frequencies greater than $0.2 \mathrm{~Hz}$. In Figure $5 b$ it is shown that significant energy transfer owing to atmospheric input takes place at these higher frequencies owing to wave breaking, near-surface turbulence, and possibly strong interactions with the long waves which are not accounted for adequately in these computations.

As in the previous case, the results for case 2, shown in Figure $5 b$, indicate that frictional dissipation dominates wave energy transformation. This is followed by refraction and shoaling effects. Because $k_{p} h$ in this case is greater than 1.0 , the nonlinear wave-wave transfer is small. It is noted, however, that further finite-depth computations into shallow water from station $5 \mathrm{~N}$ to shore would yield much higher nonlinear wave-wave transfers as $k_{p} h$ approaches 0.5 (see Figure 3).
For both cases 1 and 2, the dissipation owing to percolation was found to be small in comparison with friction because of the medium to fine sand found between stations $8 \mathrm{~N}$ and $5 \mathrm{~N}$.

\section{Summary of Relative Significance of Different Processes}

We have examined wave-energy dissipation rates in finitedepth water calculated from measured wave spectra at different stations in the offshore direction. A variety of processes have been suggested as the cause of wave attenuation in finite-depth water, and we conclude that most of these processes can indeed become important in particular situations. The nonlinear transfer by wave-wave interaction, which has been neglected in most finite-depth models, is found to be important in very shallow water defined $k_{p} h \approx 0.5$. The relative significance of friction, percolation, and bottom motion depends strongly on the type of sediment found in an area and the local currents. These are therefore important inputs for identifying the dominant dissipation mechanism operating at a site.

The relative significance of the mechanisms governing wave transformation in finite-depth water are summarized in Figure 6. Each mechanism depends on $k_{p} h$, where $k_{p}$ is the wave number of the dominant wave approaching shore and on additional parameters pertinent to that mechanism. The nonlinear wave-wave transfer is shown in Figure $6 a$ and depends most strongly on the equilibrium range level $\alpha^{\prime}$ in addition to
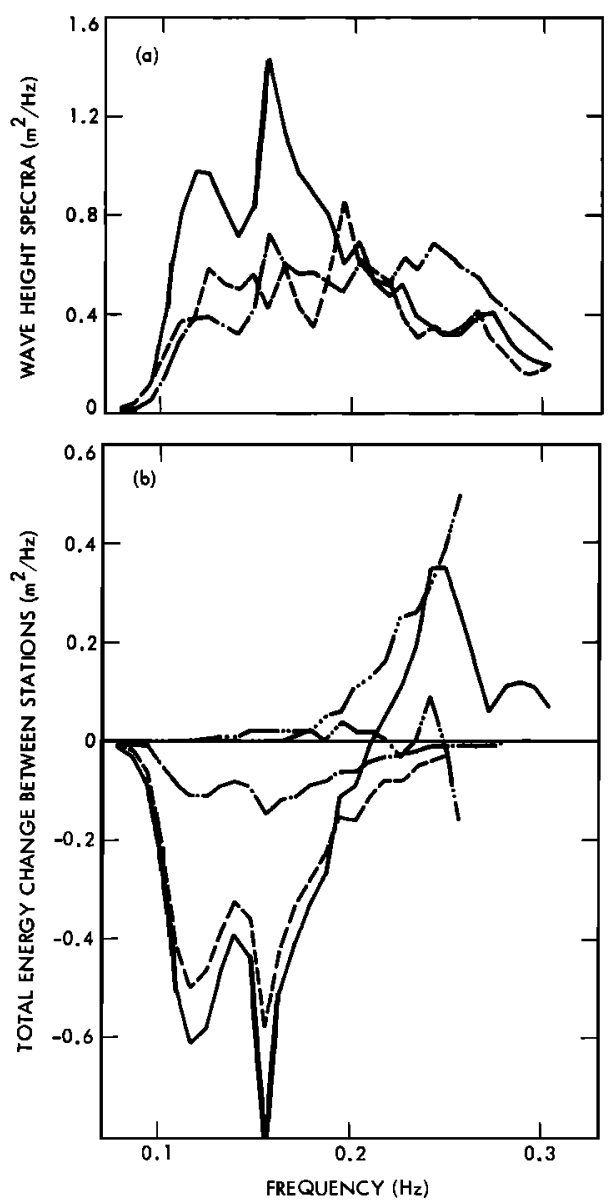

Fig. 5. Wave transformation computations in finite-depth water for case 2, August 23, 1975, at 2000 GMT. Notations for Figures $5 a$ and $5 b$ are as in Figure 4. 


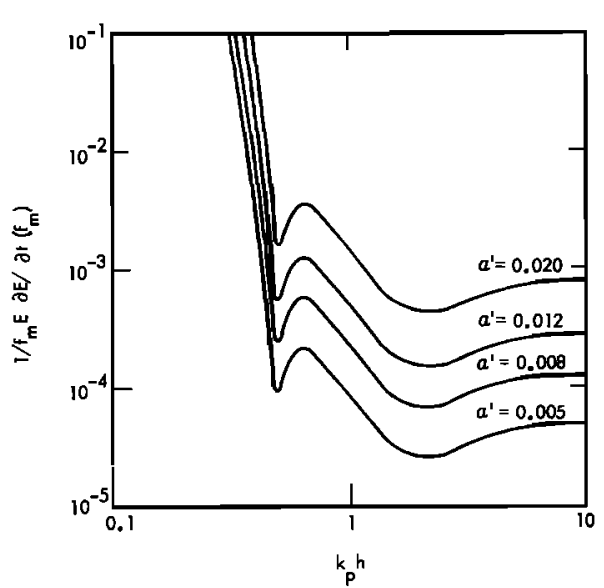

(a) WAVE - WAVE INTERACTION TRANSFER RATE AT THE SPECTRAL PEAK AS A FUNCTION OF THE EQUILIBRIUM RANGE LEVEL $a^{\prime}$,

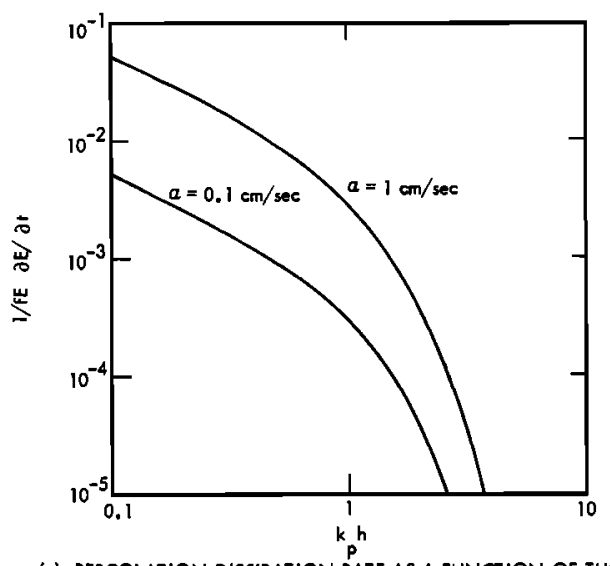

(c) PERColation dissipation RATE as a function OF the PERMEABILITY COEFFICENT, a (ISOTROPIC SAND PROPERT IES ARE ASSUMED). THE DISSIPATION RATE ALSO DEPENDS ON WAVE PERIOD, $T$ ( $T=7.75$ \& USED IN THIS FIGURE).

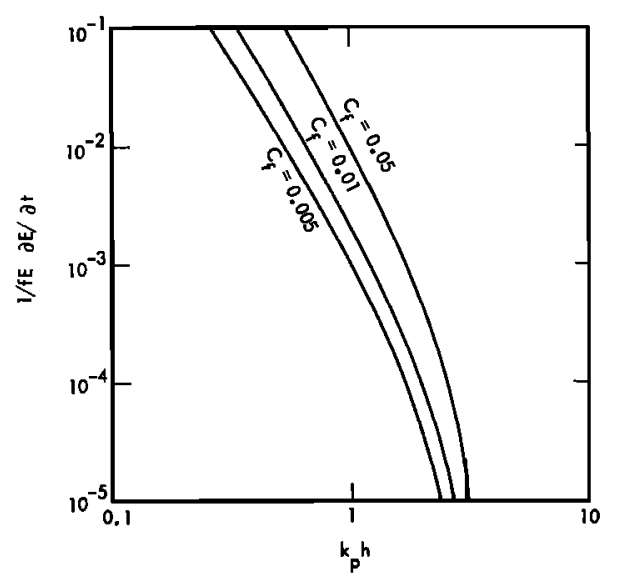

(b) ENERGY DISSIPATION RATE DUE TO FRICTION AS A FUNCTION OF $C_{f}$. IHE DISSIPATION RATE ALSO DEPENDS ON WAVE HEIGHT, $H$ AND PERIOD, $T$ ( $H=2.0 \mathrm{~m}$ AND $\mathrm{T}=7.75$ s USED IN THIS FIGURE).

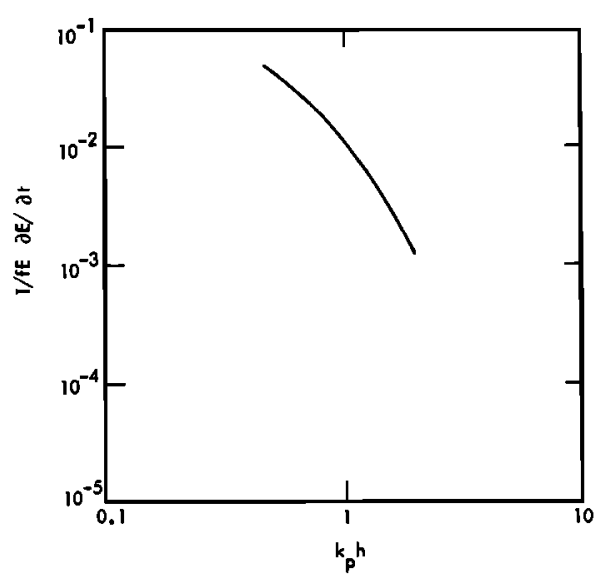

(d) BOTTOM MOTION DISSIPATION RATE FOR MUD VISCOSITY $\nu=0.6 \mathrm{~m}^{2} / \mathrm{s}$, RATIO OF MUD SHEAR MODULUS TO DENSITY $G / \rho=10 \mathrm{~m} 2 / \mathrm{s}^{2}$, MUD THICKNESS $=3 \mathrm{~m}$, AND WAVE PERIOD $=7.75 \mathrm{l}$.

Fig. 6. Relative significance of wave transformation mechanisms as a function of $k_{p} h$.

$k_{p} h$ [Herterich and Hasselmann, 1980]. The friction dissipation rate, displayed in Figure $6 b$, depends on the additional parameters $C_{f}$ (which is dependent on bottom sediments), wave height, and wave period [Hsiao and Shemdin, 1978]. The percolation dissipation rate, shown in Figure $6 c$, depends on the permeability coefficients. Finally, the bottom motion dissipation rate in Figure $6 d$ depends on the elasticity and the viscosity properties of the soft bottom sediment in addition to $k_{p} h$ [Hsiao and Shemdin, 1980]. All the graphs in Figure 6 are displayed to the same scale to allow comparisons in magnitudes of dissipation rates contributed by the different mechanisms.

Acknowledgment. Financial support for this study was provided by ONR-Geography Program, contract N00014-76-MP60029 and by NATO special grant SRG 14. K. Richter of the Deutsches Hydrographisches Institut provided useful information on sand properties in the area offshore of Sylt. This paper presents the results of one phase of research carried out at the Jet Propulsion Laboratory, California Institute of Technology, under contract NAS7-100, sponsored by the National Aeronautics and Space Administration.

\section{REFERENCES}

Bretschneider, C. L., and R. O. Reid, Changes in wave height due to bottom friction, percolation, and refraction, Tech. Memo. 45, Beach Erosion Board, U.S. Army Corps of Engineers, Washington, D. C., 1958.
Dingler, J. R., Wave-formed ripples in nearshore sands, Ph.D. dissertation, University of California, San Diego, 1974.

Fox, M. J. H., On the nonlinear transfer of energy in the peak of a gravity-wave spectrum II, Proc. Roy. Soc., London A, 348, 467-483, 1976.

Hasselmann K., and J. I. Collins, Spectral dissipation of finite-depth gravity waves due to turbulent bottom friction, J. Marine Res., 26, 1-12, 1968.

Hasselmann, S., and K. Hasselmann, A symmetrical method of computing the nonlinear transfer in a gravity wave spectrum, report, Max-Planck-Inst. für Meteorol., Hamburg, Germany, 1980.

Hasselmann, K., T. P. Barnett, E. Bouws, H. Carlson, D. E. Cartwright, K. Enke, J. A. Ewing, H. Gienapp, D. E. Hasselmann, P. Kruseman, A. Meerburg, P. Muller, D. J. Olbers, K. Richter, W. Sell, and $H$. Walden, Measurements of wind-wave growth and swell decay during the Joint North Sea Wave Project (JONSWAP), Deut. Hydrogr. Z., A, 12, 1973.

Herterich, $K$., and K. Hasselmann, On the effect of finite-depth on the nonlinear energy transfer in a surface-wave spectrum, $J$. Fluid Mech., in press, 1980.

Hsiao, S. V., and O. H. Shemdin, Bottom dissipation in finite-depth water waves, Proc. 16th Int. Conf. Coast. Eng., 1978.

Hsiao, S. V., and O. H. Shemdin, Interaction of ocean waves with a soft bottom, J. Phys. Oceanogr., in press, 1980.

Jonsson, I. G., Friction factor diagrams for oscillatory boundary layers, Basic Res. Prog. 10, Tech. Univ. of Denmark, Copenhagen, 1965.

Kajiura, K., and R. O. Reid, On the damping of gravity waves over a permeable sea bed, Eos Trans. $A G U, 38(5), 662,1957$. 
Kamphuis, J. W., Friction factor under oscillatory waves, Proc. Amer. Soc. Civil Eng., 101(WW2), 135-144, 1975.

Long, R. B., Scattering of surface waves by an irregular bottom, $J$. Geophys. Res., 78(33), 7861-7870, 1973.

Longuet-Higgins, $M$. S., On the nonlinear transfer of energy in the peak of a gravity-wave spectrum: A simplified model, Proc. Roy. Soc. London A, 347, 311-328, 1976.

Longuet-Higgins, M. S., On the dynamics of steep gravity waves in deep water, in Turbulent Fluxes Through the Sea Surface, Wave Dynamics, and Prediction, pp. 199-220, Plenum, New York, 1978.

Putnam, J. A., Loss of wave energy due to percolation in a permeable sea bottom, Eos Trans. AGU, 3, 349-356, 1949.

Richter, K., B. Schmalfeldt, and J. Siebert, Bottom irregularities in the North Sea, Deut. Hydrogr. Z., 29, 1-10, 1976.

Rosenthal, W., Energy exchange between surface waves and motion of sediment, J. Geophys. Res., 83(C4), 1980-1983, 1978.

Savage, R. P., Laboratory study of wave energy loss by bottom friction and percolation, Tech. Memo. 31, Beach Erosion Board, U.S. Army Corps of Engineers, Washington, D. C., 1953.
Shemdin, O. H., K. Hasselmann, S. V. Hsiao, and K. Herterich, Nonlinear and linear bottom interaction effects in shallow water, in Turbulent Fluxes through the Sea Surface, Wave Dynamics, and Prediction, pp. 347-372, Plenum, New York, 1978.

Sleath, J. F. H., Wave-Induced Pressures in Beds of Sand, J. Hydrol. Div., HY2, 367-378, 1970.

Tubman, M. W., and Suhayda, J. N., Wave action and bottom movements in fine sediments, Proc. 15th Int. Conf. on Coast. Eng., 1168 $1183,1976$.

Tunstall, E. B., and Inman, D. L., Vortex generation by oscillatory flow over rippled surfaces, J. Geophys. Res., 8O(24), 3475-3484, 1975.

(Received July 11, 1979;

revised October 19, 1979,

accepted April 4, 1980.) 